\title{
Willingness to Use Home Collection Methods to Provide Specimens for SARS-CoV-2/COVID-19 Research: Survey Study
}

Eric William Hall ${ }^{1}$, MPH, PhD; Nicole Luisi ${ }^{1}$, MPH, MS; Maria Zlotorzynska ${ }^{1}$, PhD, MPH; Gretchen Wilde ${ }^{1}$, MPH; Patrick Sullivan ${ }^{1}$, PhD, DVM; Travis Sanchez ${ }^{1}$, MPH, DVM; Heather Bradley ${ }^{2}$, MHS, PhD; Aaron J Siegler ${ }^{3}$, MHS, $\mathrm{PhD}$

${ }^{1}$ Department of Epidemiology, Rollins School of Public Health, Emory University, Atlanta, GA, United States

${ }^{2}$ Department of Epidemiology \& Biostatistics, School of Public Health, Georgia State University, Atlanta, GA, United States

${ }^{3}$ Department of Behavioral, Social and Health Education Sciences, Rollins School of Public Health, Emory University, Atlanta, GA, United States

\section{Corresponding Author:}

Aaron J Siegler, MHS, PhD

Department of Behavioral, Social and Health Education Sciences

Rollins School of Public Health

Emory University

1518 Clifton Road

Atlanta, GA, 30322

United States

Phone: 14047129733

Email: asiegle@emory.edu

\section{Abstract}

Background: Innovative laboratory testing approaches for SARS-CoV-2 infection and immune response are needed to conduct research to establish estimates of prevalence and incidence. Self-specimen collection methods have been successfully used in HIV and sexually transmitted infection research and can provide a feasible opportunity to scale up SARS-CoV-2 testing for research purposes.

Objective: The aim of this study was to assess the willingness of adults to use different specimen collection modalities for themselves and children as part of a COVID-19 research study.

Methods: Between March 27 and April 1, 2020, we recruited 1435 adults aged 18 years or older though social media advertisements. Participants completed a survey that included 5-point Likert scale items stating how willing they were to use the following specimen collection testing modalities as part of a research study: home collection of a saliva sample, home collection of a throat swab, home finger-prick blood collection, drive-through site throat swab, clinic throat swab, and clinic blood collection. Additionally, participants indicated how the availability of home-based collection methods would impact their willingness to participate compared to drive-through and clinic-based specimen collection. We used Kruskal-Wallis tests and Spearman rank correlations to assess if willingness to use each testing modality differed by demographic variables and characteristics of interest. We compared the overall willingness to use each testing modality and estimated effect sizes with Cohen $d$.

Results: We analyzed responses from 1435 participants with a median age of $40.0(\mathrm{SD}=18.2)$ years and over half of which were female (761/1435, 53.0\%). Most participants agreed or strongly agreed that they would be willing to use specimens self-collected at home to participate in research, including willingness to collect a saliva sample $(1259 / 1435,87.7 \%)$ or a throat swab (1191/1435, $83.1 \%)$. Willingness to collect a throat swab sample was lower in both a drive-through setting (64\%) and clinic setting (53\%). Overall, 69.0\% (990/1435) of participants said they would be more likely to participate in a research study if they could provide a saliva sample or throat swab at home compared to going to a drive-through site; only 4.4\% (63/1435) of participants said they would be less likely to participate using self-collected samples. For each specimen collection modality, willingness to collect specimens from children for research was lower than willingness to use on oneself, but the ranked order of modalities was similar.

Conclusions: Most participants were willing to participate in a COVID-19 research study that involves laboratory testing; however, there was a strong preference for home specimen collection procedures over drive-through or clinic-based testing. To increase participation and minimize bias, epidemiologic research studies of SARS-CoV-2 infection and immune response should consider home specimen collection methods. 
(J Med Internet Res 2020;22(9):e19471) doi: 10.2196/19471

\section{KEYWORDS}

COVID-19; SARS-CoV-2; specimen collection; survey; research; public health; infectious disease; virus; test

\section{Introduction}

The first case of the novel coronavirus SARS-CoV-2 in the United States was identified on January 20, 2020 [1]. By April 8 , the number of reported cases in the United States had surpassed 400,000 [2]. Over that same time period, roughly 2 million specimens had been tested for SARS-CoV-2 RNA in laboratories across the country [3]. While increased laboratory capacity and the opening of drive-through facilities has increased testing access and case identification, these strategies remain insufficient to support the population-based research required to characterize the epidemiologic nature of this outbreak because testing is focused in clinical settings and on people with symptoms of disease. To develop a better understanding of the exposure, disease, and recovery process associated with SARS-CoV-2 infection, infectious disease researchers have called for innovative testing approaches and a rapid scaleup in the number of persons tested [4].

Self-specimen collection for testing has been successfully used in HIV and sexually transmitted infection (STI) research for well over a decade [5-8]. A review of 25 HIV testing studies found that across multiple specimen methods (finger prick, oral swabs), self-collection results had the same diagnostic accuracy as clinician-collected specimens, with no differences in the proportions of invalid results [9]. Another review comparing participant self-collection versus clinician collection for gonorrhea and chlamydia also found high performance (>90\% sensitivity and specificity) for self-collected specimens [10]. In one of our previous studies, $93 \%$ of participants were able to successfully complete multiple specimen collections, and $85 \%$ preferred self-collection of specimens at home to a standard office visit [11]. In the present analysis, we aimed to assess the willingness of adults to use different specimen collection methods on themselves or their children as part of a COVID-19 research study. We hypothesized that modalities for home specimen collection would be preferred over clinic-based specimen collection.

\section{Methods}

\section{Recruitment}

Participants were recruited through web-based social media advertisements on Facebook, Snapchat, and Twitter from March 27, 2020 to April 1, 2020. Internet users who clicked on the advertisements were taken to a consent module and short screener to determine eligibility. Eligible respondents were adults aged $\geq 18$ years. On the last day of recruitment, we oversampled Hispanic and Black respondents with targeted ads to increase the racial and ethnic diversity of the sample. Eligible participants completed a web-based survey that collected data on their demographics, current knowledge of COVID-19, stigma related to COVID-19, and relevant symptoms over the last 24 hours. We used cookie-based duplicate protection, which restricts respondents from completing the survey more than once from the same browser on the same device. Participants were not compensated for their participation.

Next, the participants answered a series of 5-point Likert scale items ( $1=$ strongly disagree, $2=$ disagree, $3=$ undecided, $4=$ agree, $5=$ strongly agree) about their willingness to use different specimen collection modalities to test for SARS-CoV-2 infection as part of a research study. Participants who indicated having children aged $<18$ years in their household were also asked about their willingness to use the same modalities to collect specimens from their child as part of a research study. The modalities included home collection of a saliva sample, home throat swab collection, home finger prick blood collection, drive-through site throat swab collection, clinic throat swab collection, and clinic blood collection. The questions indicated that all specimens collected at home would be mailed to a central laboratory for testing. The definitions provided to participants for each testing modality are reported in Table 1.

Finally, the participants were asked how the availability of a home specimen collection method to test for COVID-19 that used either a saliva sample or throat swab would impact their willingness to participate in a research study compared to a drive-through sample collection site and a clinic sample site. For these questions, possible answers included "more likely to participate in a research study," "about the same likelihood to participate in a research study," and "less likely to participate in a research study." 
Table 1. Definitions of specimen collection testing modalities used in a web-based survey to assess willingness to participate in a COVID-19 research study in the United States in March 2020.

\begin{tabular}{ll}
\hline Testing modality & Survey definition \\
\hline $\begin{array}{l}\text { Home saliva sample } \\
\text { Home throat swab }\end{array}$ & $\begin{array}{l}\text { A home saliva sample would involve you spitting in a tube and sending it to a certified laboratory. } \\
\text { A home throat swab would involve you using a throat swab and sending it to a certified laboratory. } \\
\text { Home blood collection }\end{array}$ \\
$\begin{array}{l}\text { A home blood test would involve using an automated finger prick device, collecting a blood sample on a specimen } \\
\text { card, and mailing in a prepaid mailer to a certified laboratory. }\end{array}$ \\
$\begin{array}{l}\text { A drive-through site throat swab } \\
\text { a health care worker collect the swab. }\end{array}$ \\
$\begin{array}{l}\text { A laboratory throat swab would involve your traveling to a laboratory facility in a clinic or private laboratory } \\
\text { to have a health care worker collect the swab. }\end{array}$ \\
Alinic blood collection & $\begin{array}{l}\text { A laboratory blood test would involve your traveling to a laboratory facility in a clinic or a private lab to have } \\
\text { blood drawn, similar to a usual doctor's visit. }\end{array}$
\end{tabular}

\section{Statistical Analysis}

All analysis was performed using RStudio v1.1.453. To present a complete description of these data, we summarized the participants' willingness to use each testing modality by calculating both the mean (SD) and median (IQR). A stigma index score was calculated by summing the number of stigma-related items the participant indicated as true (maximum=4). Similarly, we calculated a knowledge index score by tabulating the number of correct responses to the knowledge items (maximum=14). In the methodological literature, there is an ongoing debate about whether parametric or nonparametric statistical methods should be used for Likert-type data [12,13]; therefore, we explored the data with both methods. First, we used nonparametric Kruskal-Wallis tests to assess if willingness to use each testing modality differed by categorical demographic variables and nonparametric Spearman rank correlation coefficients to assess if willingness differed by ordinal characteristics (eg, income, education, likelihood of currently having COVID-19, stigma index score, knowledge index score, and number of symptoms in the past 24 hours). Second, we used parametric statistical methods to facilitate interpretation of the main findings, using Cohen $d$ to estimate the effect size of the overall willingness to use each testing modality. Cohen $d$ reports the estimated difference in mean values in terms of SD [14]. For example, a Cohen $d$ of 1 indicates that the mean of one group is one standard deviation away from than the mean of the comparison group. All $P$ values were adjusted for multiple tests using the Bonferroni-Holm method.

\section{Results}

A total of 4593 respondents started the eligibility screener. Of these, $12(0.3 \%)$ were removed for duplicate IP addresses, 1260 (27.4\%) did not meet the eligibility criteria, and 1886 (41.1\%) failed to complete the primary outcome survey questions, resulting in an analytic dataset of 1435 (31.2\%) survey responses. The demographic characteristics are summarized in Table 2. Over half the participants $(761 / 1435,53.0 \%)$ were female, and the mean age was 40.0 years (SD 18.2). Many participants were non-Hispanic White $(587 / 1435,40.9 \%)$ or Hispanic (548/1435, 38.2\%), and most had either completed a college degree $(629 / 1435,43.8 \%)$ or attended some college, associate degree, or technical school $(382 / 1435,26.6 \%)$. Over one-quarter of respondents $(385 / 1435,26.8 \%)$ reported children aged $<18$ years in their household and answered survey questions about their willingness to use different specimen collection modalities for SARS-CoV-2 testing to collect specimens from their children.

Figure 1 displays the participants' stated willingness to use different specimen collection modalities for SARS-CoV-2 testing on themselves and their children. Overall, the large majority of participants agreed or strongly agreed that they would be willing to use a home specimen collection method to obtain a saliva sample $(1259 / 1435,87.7 \%)$ or a throat swab $(1191 / 1435,83.1 \%)$ from themselves as part of a research study. More than half the participants agreed or strongly agreed that they would be willing to acquire a home specimen collection finger prick blood sample (928/1435, 64.7\%), visit a drive-through site to provide a throat swab $(914 / 1435,63.7 \%)$, or visit a clinic to provide a blood sample $(812 / 1435,56.6 \%)$ or a throat swab $(762 / 1435,53.1 \%)$. In a separate question about relative preference between multiple specimen collection modalities, $990 / 1435$ participants $(69.0 \%)$ said they would be more likely to participate in a research study if they could collect a saliva sample or throat swab at home compared to going to a drive-through site. Similarly, 1023/1435 participants (71.3\%) stated that they would be more likely to participate in a research study with specimens to be collected at home compared to a study with specimens collected at a clinic. Of the 1435 participants, only $63(4.4 \%)$ and $82(5.7 \%)$ reported that using a home specimen collection process would make them less likely to participate in a research study compared to sample collection at a drive-through site or a clinic, respectively.

Relative to the participants' willingness to participate in research themselves, their willingness to have their children participate in research was lower for each specimen collection modality (Figure 1). The proportion of participants willing to use each modality to collect specimens from their children ranged from $291 / 385(75.6 \%)$ who were willing to perform home collection of a saliva sample to $124 / 334(37.1 \%)$ who were willing to take their child to a clinic for a blood sample. However, the ranked orders of the participants' willingness to use each testing modality on themselves and on their children were similar. 
For most comparisons, the stated willingness to use each specimen collection modality did not differ by demographic group, stigma index score, or presence of current COVID-19 symptoms (Table 2, Table 3, and Table 4). A notable exception was that younger participants were slightly less willing to obtain a home-collected throat swab (adjusted $P=.049$ ) or visit a drive-through site to provide a throat swab (adjusted $P=.047$ ).
While there was no difference in willingness to use home collection saliva samples or throat swabs, participants who thought it was somewhat likely, likely, or very likely that they currently had COVID-19 had moderately higher willingness to visit a drive-through site (adjusted $P=.01$ ) or a clinic (adjusted $P=.003$ ) to provide a throat swab. 
Table 2. Demographic characteristics of the study participants (internet-using adults aged $>18$ years in the United States in March 2020) and their stated willingness to use home saliva sample and throat swab specimen collection testing modalities on themselves as part of a COVID-19 research study $(\mathrm{N}=1435)$. All survey questions were 5 -point Likert scale items where $1=$ strongly disagree, $2=$ disagree, $3=$ undecided, $4=$ agree, $5=$ strongly agree Kruskal-Wallis tests and Spearman rank correlation coefficients were used to assess response differences by characteristic.

\begin{tabular}{|c|c|c|c|c|c|c|c|}
\hline \multirow[t]{2}{*}{ Characteristic } & \multirow[t]{2}{*}{$\mathrm{n}(\%)$} & \multicolumn{3}{|c|}{ Home: saliva sample } & \multicolumn{3}{|c|}{ Home: throat swab } \\
\hline & & Mean (SD) & Median (IQR) & $P$ value $^{\mathrm{a}}$ & Mean (SD) & Median (IQR) & $P$ value \\
\hline Overall & $1435(100.0)$ & $4.5(0.9)$ & $5(4-5)$ & $\mathrm{N} / \mathrm{A}^{\mathrm{b}}$ & $4.4(1.9)$ & $5(4-5)$ & N/A \\
\hline Gender & & & & $>.99$ & & & $>.99$ \\
\hline Female & $761(53.0)$ & $4.5(0.9)$ & $5(4-5)$ & & $4.3(1.0)$ & $5(4-5)$ & \\
\hline Male & $536(37.4)$ & $4.5(0.9)$ & $5(4-5)$ & & $4.4(1.0)$ & $5(4-5)$ & \\
\hline Other & $36(2.5)$ & $4.6(1.0)$ & $5(5-5)$ & & $4.5(1.0)$ & $5(4.75-5)$ & \\
\hline Age (years) & & & & .14 & & & .049 \\
\hline $18-29$ & $560(39.0)$ & $4.4(1.0)$ & $5(4-5)$ & & $4.2(1.1)$ & $5(4-5)$ & \\
\hline $30-49$ & $391(27.2)$ & $4.5(0.9)$ & $5(4-5)$ & & $4.4(1.0)$ & $5(4-5)$ & \\
\hline $50-64$ & $289(20.1)$ & $4.6(0.7)$ & $5(4-5)$ & & $4.5(0.9)$ & $5(4-5)$ & \\
\hline$\geq 65$ & $194(13.5)$ & $4.5(0.9)$ & $5(4-5)$ & & $4.4(1.0)$ & $5(4-5)$ & \\
\hline Race/ethnicity & & & & .06 & & & $>.99$ \\
\hline Hispanic & $548(38.2)$ & $4.5(0.9)$ & $5(4-5)$ & & $4.4(1.0)$ & $5(4-5)$ & \\
\hline Asian/Pacific Islander & $52(3.6)$ & $4.6(0.7)$ & $5(4-5)$ & & $4.5(0.8)$ & $5(4-5)$ & \\
\hline Non-Hispanic Black & $158(11.0)$ & $4.3(1.0)$ & $5(4-5)$ & & $4.2(1.1)$ & $5(4-5)$ & \\
\hline Non-Hispanic White & $587(40.9)$ & $4.6(0.9)$ & $5(4-5)$ & & $4.4(1.0)$ & $5(4-5)$ & \\
\hline Other & $90(6.2)$ & $4.3(1.1)$ & $5(4-5)$ & & $4.1(1.3)$ & $5(4-5)$ & \\
\hline Education & & & & $>.99$ & & & $>.99$ \\
\hline $\begin{array}{l}\text { College, postgraduate, or profession- } \\
\text { al school }\end{array}$ & $629(43.8)$ & $4.5(0.9)$ & $5(4-5)$ & & $4.4(1.0)$ & $5(4-5)$ & \\
\hline $\begin{array}{l}\text { Some college, associate's degree, } \\
\text { or technical school }\end{array}$ & $382(26.6)$ & $4.6(0.8)$ & $5(4-5)$ & & $4.5(0.9)$ & $5(4-5)$ & \\
\hline High school/GED ${ }^{c}$ & $175(12.2)$ & $4.4(1.0)$ & $5(4-5)$ & & $4.3(1.1)$ & $5(4-5)$ & \\
\hline Did not finish high school & $27(1.9)$ & $4.6(0.9)$ & $5(4-5)$ & & $4.4(1.0)$ & $5(4-5)$ & \\
\hline Annual income (US \$) & & & & .44 & & & $>.99$ \\
\hline$<24,000$ & $294(20.5)$ & $4.5(0.9)$ & $5(4-5)$ & & $4.3(1.1)$ & $5(4-5)$ & \\
\hline 24,000 to $<50,000$ & $276(19.2)$ & $4.5(1.0)$ & $5(4-5)$ & & $4.4(1.1)$ & $5(4-5)$ & \\
\hline 50,000 to $<75,000$ & $203(14.1)$ & $4.6(0.9)$ & $5(4-5)$ & & $4.5(0.9)$ & $5(4-5)$ & \\
\hline$\geq 75,000$ & $268(18.7)$ & $4.7(0.8)$ & $5(5-5)$ & & $4.5(0.9)$ & $5(4-5)$ & \\
\hline Don't know & $91(6.3)$ & $4.5(0.8)$ & $5(4-5)$ & & $4.3(0.9)$ & $5(4-5)$ & \\
\hline \multicolumn{4}{|c|}{ How likely do you think it is you have COVID-19 now? } & $>.99$ & & & $>.99$ \\
\hline Very unlikely & $356(24.8)$ & $4.4(1.1)$ & $5(4-5)$ & & $4.3(1.2)$ & $5(4-5)$ & \\
\hline Unlikely & $661(46.1)$ & $4.5(0.9)$ & $5(4-5)$ & & $4.3(1.0)$ & $5(4-5)$ & \\
\hline Somewhat likely & $324(22.6)$ & $4.5(0.8)$ & $5(4-5)$ & & $4.5(0.9)$ & $5(4-5)$ & \\
\hline Likely/very likely & $81(5.6)$ & $4.5(0.8)$ & $5(4-5)$ & & $4.5(0.9)$ & $5(4-5)$ & \\
\hline Stigma index score & & & & .70 & & & $>.99$ \\
\hline 0 & $722(50.3)$ & $4.5(0.9)$ & $5(4-5)$ & & $4.4(1.0)$ & $5(4-5)$ & \\
\hline $1-2$ & $525(36.6)$ & $4.5(1.0)$ & $5(4-5)$ & & $4.4(1.0)$ & $5(4-5)$ & \\
\hline$\geq 3$ & $106(7.4)$ & $4.3(1.1)$ & $5(4-5)$ & & $4.3(1.2)$ & $5(4-5)$ & \\
\hline Knowledge index score & & & & .02 & & & $>.99$ \\
\hline
\end{tabular}




\begin{tabular}{|c|c|c|c|c|c|c|c|}
\hline \multirow[t]{2}{*}{ Characteristic } & \multirow[t]{2}{*}{$\mathrm{n}(\%)$} & \multicolumn{3}{|c|}{ Home: saliva sample } & \multicolumn{3}{|c|}{ Home: throat swab } \\
\hline & & Mean (SD) & Median (IQR) & $P$ value $^{\mathrm{a}}$ & Mean (SD) & Median (IQR) & $P$ value \\
\hline$<12$ & $337(23.5)$ & $4.4(0.9)$ & $5(4-5)$ & & $4.4(1.0)$ & $5(4-5)$ & \\
\hline $12-13$ & $655(45.6)$ & $4.5(1.0)$ & $5(4-5)$ & & $4.4(1.1)$ & $5(4-5)$ & \\
\hline 14 & $342(23.8)$ & $4.5(0.9)$ & $5(4-5)$ & & $4.4(1.0)$ & $5(4-5)$ & \\
\hline Symptoms & & & & .71 & & & $>.99$ \\
\hline 1 or more symptoms & $747(52.1)$ & $4.5(0.9)$ & $5(4-5)$ & & $4.4(1.0)$ & $5(4-5)$ & \\
\hline None & 688 (47.9) & $4.4(1.0)$ & $5(4-5)$ & & $4.3(1.1)$ & $5(4-5)$ & \\
\hline
\end{tabular}

${ }^{\mathrm{a}} P$ values were adjusted for multiple comparisons using the Bonferroni-Holm method.

b/A: not applicable.

${ }^{\mathrm{c}}$ GED: General Education Development.

Figure 1. Stated willingness to use testing modalities as part of a COVID-19 research study by internet-using adults aged $\geq 18$ years in the United States in March 2020 on themselves (top) and on their children (bottom). All specimen collection modalities involved testing the specimens in a central laboratory.

\section{Willingness to use specimen collection modalities on self to test for COVID-19 as part of a research study}

\begin{tabular}{|c|c|c|c|c|c|}
\hline \multirow[b]{2}{*}{ At-home: Saliva Sample } & \multicolumn{5}{|c|}{$(n=1435)$} \\
\hline & $5 \%$ & & $7 \%$ & & $88 \%$ \\
\hline At-home: Throat Swab & $7 \%$ & & $10 \%$ & & $83 \%$ \\
\hline At-home: Blood Sample & $18 \%$ & & $17 \%$ & & $65 \%$ \\
\hline Drive-through Site: Throat Swab & $16 \%$ & & $20 \%$ & & $64 \%$ \\
\hline Clinic: Blood Sample & $22 \%$ & & $22 \%$ & & $57 \%$ \\
\hline Clinic: Throat Swab & $25 \%$ & & $22 \%$ & & $53 \%$ \\
\hline & 100 & 50 & $\begin{array}{c}0 \\
\text { centa }\end{array}$ & 50 & 100 \\
\hline
\end{tabular}

Willingness to use specimen collection modalities on children to test for COVID-19 as part of a research study

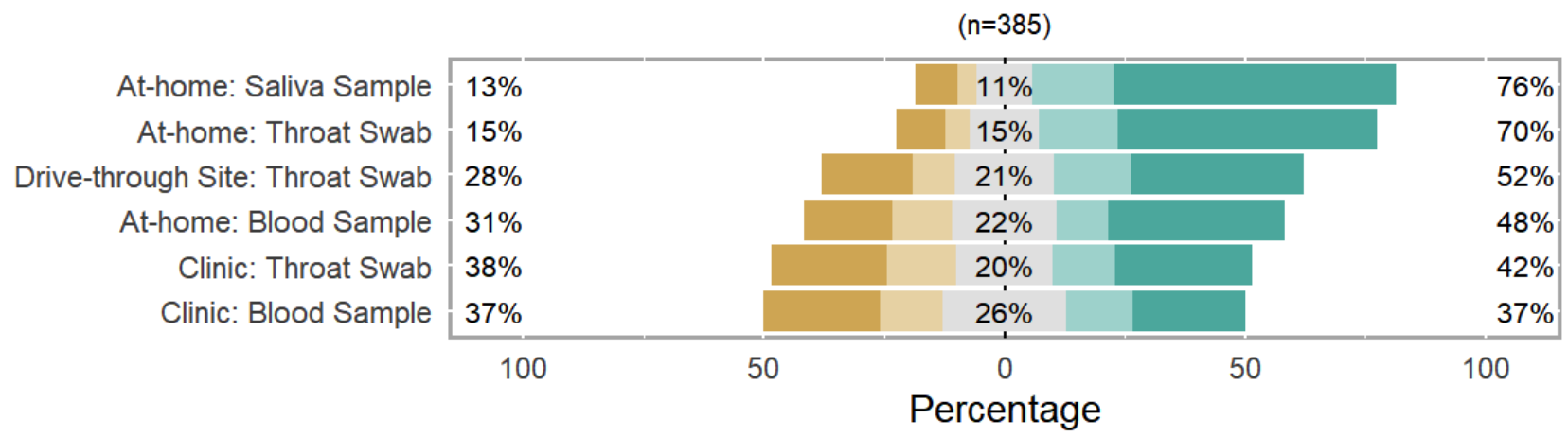

\begin{tabular}{l|l|l|l|l|l} 
Response & Strongly Disagree & Disagree & Undecided & Agree & Strongly Agree
\end{tabular} 
Table 3. Stated willingness of internet-using adults aged $\geq 18$ years in the United States in March 2020 to use drive-through and clinic throat swab specimen collection testing modalities on themselves as part of a COVID-19 research study ( $\mathrm{N}=1435)$. All survey questions were 5-point Likert scale items where $1=$ strongly disagree, $2=$ disagree, $3=$ undecided, $4=$ agree, $5=$ strongly agree. Kruskal-Wallis tests and Spearman rank correlation coefficients were used to assess response differences by characteristic.

\begin{tabular}{|c|c|c|c|c|c|c|}
\hline \multirow[t]{2}{*}{ Characteristic } & \multicolumn{3}{|c|}{ Drive-through site: throat swab } & \multicolumn{3}{|c|}{ Clinic: throat swab } \\
\hline & Mean (SD) & Median (IQR) & $P$ value $^{\mathrm{a}}$ & Mean (SD) & Median (IQR) & $P$ value \\
\hline Overall & $3.8(1.3)$ & $4(3-5)$ & $\mathrm{N} / \mathrm{A}^{\mathrm{b}}$ & $3.5(1.4)$ & $4(3-5)$ & N/A \\
\hline Gender & & & $>.99$ & & & .57 \\
\hline Female & $3.8(1.3)$ & $4(3-5)$ & & $3.4(1.4)$ & $4(2-5)$ & \\
\hline Male & $3.9(1.3)$ & $4(3-5)$ & & $3.6(1.4)$ & $4(3-5)$ & \\
\hline Other & $3.7(1.4)$ & $4(3-5)$ & & $3.6(1.2)$ & $3.5(3-5)$ & \\
\hline Age (years) & & & .047 & & & $>.99$ \\
\hline $18-29$ & $3.7(1.4)$ & $4(3-5)$ & & $3.5(1.4)$ & $4(3-5)$ & \\
\hline $30-49$ & $3.9(1.3)$ & $4(3-5)$ & & $3.5(1.4)$ & $4(3-5)$ & \\
\hline $50-64$ & $4(1.3)$ & $4(3-5)$ & & $3.4(1.4)$ & $4(2-5)$ & \\
\hline$\geq 65$ & $3.9(1.2)$ & $4(3-5)$ & & $3.6(1.3)$ & $4(3-5)$ & \\
\hline Race/ethnicity & & & $>.99$ & & & .99 \\
\hline Hispanic & $3.9(1.3)$ & $4(3-5)$ & & $3.6(1.4)$ & $4(3-5)$ & \\
\hline Asian/Pacific Islander & $3.7(1.4)$ & $4(3-5)$ & & $3.6(1.4)$ & $4(2.75-5)$ & \\
\hline Non-Hispanic Black & $3.7(1.4)$ & $4(3-5)$ & & $3.5(1.5)$ & $4(2-5)$ & \\
\hline Non-Hispanic White & $3.9(1.3)$ & $4(3-5)$ & & $3.4(1.4)$ & $3(2-5)$ & \\
\hline Other & $3.6(1.4)$ & $4(3-5)$ & & $3.4(1.4)$ & $3(2.25-5)$ & \\
\hline Education & & & .69 & & & $>.99$ \\
\hline $\begin{array}{l}\text { College, postgraduate, } \\
\text { or professional school }\end{array}$ & $3.8(1.3)$ & $4(3-5)$ & & $3.4(1.4)$ & $4(2-5)$ & \\
\hline $\begin{array}{l}\text { Some college, associate's degree, } \\
\text { or technical school }\end{array}$ & $4(1.2)$ & $5(3-5)$ & & $3.6(1.4)$ & $4(3-5)$ & \\
\hline High school/GED ${ }^{c}$ & $3.6(1.4)$ & $4(3-5)$ & & $3.5(1.4)$ & $4(3-5)$ & \\
\hline Did not finish high school & $3.8(1.3)$ & $4(3-5)$ & & $3.5(1.5)$ & $4(2.5-5)$ & \\
\hline Annual income (US \$) & & & $>.99$ & & & .82 \\
\hline$<24,000$ & $3.8(1.3)$ & $4(3-5)$ & & $3.7(1.4)$ & $4(3-5)$ & \\
\hline 24,000 to $<50,000$ & $3.9(1.3)$ & $4(3-5)$ & & $3.5(1.4)$ & $4(3-5)$ & \\
\hline 50,000 to $<75,000$ & $3.9(1.2)$ & $4(3-5)$ & & $3.5(1.4)$ & $4(3-5)$ & \\
\hline$\geq 75,000$ & $3.9(1.3)$ & $5(3-5)$ & & $3.4(1.5)$ & $3(2-5)$ & \\
\hline Don't know & $3.6(1.3)$ & $4(3-5)$ & & $3.4(1.3)$ & $3(3-5)$ & \\
\hline \multicolumn{3}{|c|}{ How likely do you think it is you have COVID-19 now? } & .001 & & & .003 \\
\hline Very unlikely & $3.6(1.5)$ & $4(3-5)$ & & $3.3(1.5)$ & $3(2-5)$ & \\
\hline Unlikely & $3.8(1.3)$ & $4(3-5)$ & & $3.5(1.4)$ & $4(3-5)$ & \\
\hline Somewhat likely & $4(1.2)$ & $4.5(3-5)$ & & $3.7(1.3)$ & $4(3-5)$ & \\
\hline Likely/very likely & $4(1.3)$ & $5(3-5)$ & & $3.7(1.4)$ & $4(3-5)$ & \\
\hline Stigma index score & & & $>.99$ & & & .70 \\
\hline 0 & $3.9(1.2)$ & $4(3-5)$ & & $3.6(1.3)$ & $4(3-5)$ & \\
\hline $1-2$ & $3.8(1.4)$ & $4(3-5)$ & & $3.4(1.4)$ & $3(2-5)$ & \\
\hline$\geq 3$ & $3.7(1.4)$ & $4(3-5)$ & & $3.5(1.5)$ & $4(2-5)$ & \\
\hline
\end{tabular}




\begin{tabular}{|c|c|c|c|c|c|c|}
\hline \multirow[t]{2}{*}{ Characteristic } & \multicolumn{3}{|c|}{ Drive-through site: throat swab } & \multicolumn{3}{|c|}{ Clinic: throat swab } \\
\hline & Mean (SD) & Median (IQR) & $P$ value $^{\mathrm{a}}$ & Mean (SD) & Median (IQR) & $P$ value \\
\hline Knowledge index score & & & $>.99$ & & & $>.99$ \\
\hline$<12$ & $3.9(1.3)$ & $4(3-5)$ & & $3.6(1.4)$ & $4(3-5)$ & \\
\hline $12-13$ & $3.8(1.3)$ & $4(3-5)$ & & $3.5(1.4)$ & $4(2-5)$ & \\
\hline 14 & $3.8(1.3)$ & $4(3-5)$ & & $3.4(1.4)$ & $4(2-5)$ & \\
\hline Symptoms & & & .21 & & & .65 \\
\hline 1 or more symptoms & $3.9(1.3)$ & $4(3-5)$ & & $3.6(1.4)$ & $4(3-5)$ & \\
\hline None & $3.7(1.4)$ & $4(3-5)$ & & $3.4(1.4)$ & $4(2-5)$ & \\
\hline
\end{tabular}

${ }^{\mathrm{a}}$ All $P$ values were adjusted for multiple comparisons using the Bonferroni-Holm method.

${ }^{\mathrm{b}} \mathrm{N} / \mathrm{A}$ : not applicable.

${ }^{\mathrm{c}}$ GED: General Education Development. 
Table 4. Stated willingness of internet-using adults aged $\geq 18$ years in the United States in March 2020 to use home and clinic blood sample specimen collection testing modalities on themselves as part of a COVID-19 research study $(\mathrm{N}=1435)$. All survey questions were 5-point Likert scale items where $1=$ strongly disagree, $2=$ disagree, $3=$ =undecided, $4=$ agree, $5=$ strongly agree. Kruskal-Wallis tests and Spearman rank correlation coefficients were used to assess response differences by characteristic.

\begin{tabular}{|c|c|c|c|c|c|c|}
\hline \multirow[t]{2}{*}{ Characteristic } & \multicolumn{2}{|c|}{ Home: blood sample } & \multirow[b]{2}{*}{$P$ value $^{\mathrm{a}}$} & \multicolumn{2}{|c|}{ Clinic: blood sample } & \multirow[b]{2}{*}{$P$ value } \\
\hline & Mean (SD) & Median (IQR) & & Mean (SD) & Median (IQR) & \\
\hline Overall & $3.8(1.3)$ & $4(3-5)$ & $\mathrm{N} / \mathrm{A}^{\mathrm{b}}$ & $3.6(1.3)$ & $4(3-5)$ & N/A \\
\hline Gender & & & $>0.99$ & & & $>0.99$ \\
\hline Female & $3.8(1.3)$ & $4(3-5)$ & & $3.5(1.3)$ & $4(3-5)$ & \\
\hline Male & $3.9(1.3)$ & $4(3-5)$ & & $3.7(1.3)$ & $4(3-5)$ & \\
\hline Other & $4.1(1.3)$ & $5(4-5)$ & & $3.6(1.2)$ & $4(3-5)$ & \\
\hline Age (years) & & & $<0.001$ & & & $>0.99$ \\
\hline $18-29$ & $3.5(1.4)$ & $4(2-5)$ & & $3.5(1.4)$ & $4(3-5)$ & \\
\hline $30-49$ & $4.0(1.2)$ & $4(3-5)$ & & $3.7(1.3)$ & $4(3-5)$ & \\
\hline $50-64$ & $4.1(1.1)$ & $5(3-5)$ & & $3.6(1.3)$ & $4(3-5)$ & \\
\hline$\geq 65$ & $4.0(1.2)$ & $4(3-5)$ & & $3.7(1.3)$ & $4(3-5)$ & \\
\hline Race/ethnicity & & & $>0.99$ & & & $>0.99$ \\
\hline Hispanic & $3.6(1.4)$ & $4(3-5)$ & & $3.7(1.2)$ & $4(3-5)$ & \\
\hline Asian/Pacific Islander & $3.8(1.3)$ & $4(3-5)$ & & $3.6(1.3)$ & $4(3-5)$ & \\
\hline Non-Hispanic Black & $3.6(1.4)$ & $4(3-5)$ & & $3.5(1.4)$ & $4(2-5)$ & \\
\hline Non-Hispanic White & $3.9(1.3)$ & $4(3-5)$ & & $3.5(1.3)$ & $4(3-5)$ & \\
\hline Other & $3.7(1.3)$ & $4(3-5)$ & & $3.6(1.3)$ & $4(3-5)$ & \\
\hline Education & & & $>0.99$ & & & $>0.99$ \\
\hline $\begin{array}{l}\text { College, postgraduate, } \\
\text { or professional school }\end{array}$ & $4.0(1.3)$ & $4(3-5)$ & & $3.6(1.3)$ & $4(3-5)$ & \\
\hline $\begin{array}{l}\text { Some college, associate's degree, or } \\
\text { technical school }\end{array}$ & $3.9(1.2)$ & $4(3-5)$ & & $3.4(1.2)$ & $4(3-4)$ & \\
\hline High school/GED ${ }^{c}$ & $3.7(1.3)$ & $4(3-5)$ & & $3.5(1.4)$ & $4(2-5)$ & \\
\hline Did not finish high school & $3.9(1.2)$ & $4(3-5)$ & & $3.7(1.3)$ & $4(3-5)$ & \\
\hline Annual income (US \$) & & & 0.01 & & & 0.65 \\
\hline$<24,000$ & $3.9(1.3)$ & $4(3-5)$ & & $3.7(1.2)$ & $4(3-5)$ & \\
\hline 24,000 to $<50,000$ & $4.0(1.2)$ & $4(3-5)$ & & $3.5(1.3)$ & $4(3-5)$ & \\
\hline 50,000 to $<75,000$ & $3.8(1.3)$ & $4(3-5)$ & & $3.7(1.3)$ & $4(3-5)$ & \\
\hline$\geq 75,000$ & $4.2(1.1)$ & $5(4-5)$ & & $3.7(1.3)$ & $4(3-5)$ & \\
\hline Don't know & $3.5(1.4)$ & $4(3-5)$ & & $3.5(1.3)$ & $3(3-5)$ & \\
\hline \multicolumn{3}{|c|}{ How likely do you think it is you have COVID-19 now? } & $>0.99$ & & & $>0.99$ \\
\hline Very unlikely & $3.9(1.3)$ & $4(3-5)$ & & $3.4(1.4)$ & $4(2-5)$ & \\
\hline Unlikely & $3.8(1.3)$ & $4(3-5)$ & & $3.6(1.3)$ & $4(3-5)$ & \\
\hline Somewhat likely & $3.8(1.3)$ & $4(3-5)$ & & $3.7(1.2)$ & $4(3-5)$ & \\
\hline Likely/very likely & $3.5(1.5)$ & $4(3-5)$ & & $3.6(1.5)$ & $4(3-5)$ & \\
\hline Stigma index score & & & $>0.99$ & & & $>0.99$ \\
\hline 0 & $3.9(1.3)$ & $4(3-5)$ & & $3.7(1.3)$ & $4(3-5)$ & \\
\hline $1-2$ & $3.8(1.4)$ & $4(3-5)$ & & $3.5(1.4)$ & $4(2-5)$ & \\
\hline$\geq 3$ & $3.7(1.4)$ & $4(2-5)$ & & $3.4(1.4)$ & $4(2-5)$ & \\
\hline Knowledge index score & & & $>0.99$ & & & $>0.99$ \\
\hline
\end{tabular}




\begin{tabular}{|c|c|c|c|c|c|c|}
\hline \multirow[t]{2}{*}{ Characteristic } & \multicolumn{3}{|c|}{ Home: blood sample } & \multicolumn{3}{|c|}{ Clinic: blood sample } \\
\hline & Mean (SD) & Median (IQR) & $P$ value $^{\mathrm{a}}$ & Mean (SD) & Median (IQR) & $P$ value \\
\hline$<12$ & $3.8(1.3)$ & $4(3-5)$ & & $3.6(1.3)$ & $4(3-5)$ & \\
\hline $12-13$ & $3.9(1.3)$ & $4(3-5)$ & & $3.6(1.3)$ & $4(3-5)$ & \\
\hline 14 & $3.8(1.3)$ & $4(3-5)$ & & $3.5(1.4)$ & $4(3-5)$ & \\
\hline Symptoms & & & $>0.99$ & & & $>0.99$ \\
\hline 1 or more symptoms & $3.8(1.3)$ & $4(3-5)$ & & $3.6(1.3)$ & $4(3-5)$ & \\
\hline None & $3.9(1.3)$ & $4(3-5)$ & & $3.5(1.3)$ & $4(3-5)$ & \\
\hline
\end{tabular}

${ }^{\text {a }} P$ values were adjusted for multiple comparisons using the Bonferroni-Holm method.

b/A: not applicable.

${ }^{\mathrm{c}}$ GED: General Education Development.

The mean willingness rating (Likert 5-point scale) ranged from 3.5 (clinic throat swab, median 4) to 4.5 (home saliva sample, median 5; Table 5). Compared to the participants' willingness to use a home saliva sample, there was a medium effect size in willingness to use a home test blood sample (Cohen $d=0.568$; 95\% CI 0.510-0.627) and willingness to use a drive-through throat swab (Cohen $d=0.567$; 95\% CI 0.507-0.627). There was a large effect size in willingness to use a clinic for either a throat swab (Cohen $d=0.802 ; 95 \%$ CI 0.732-0.872) or a blood sample (Cohen $d=0.776$; 95\% CI 0.706-0.847) compared to using a home test saliva sample. A similar pattern was seen in comparisons between willingness to use different testing modalities for children.

Table 5. Stated willingness of internet-using adults aged $\geq 18$ years in the United States in March 2020 to use specimen collection modalities on themselves and their children as part of a COVID-19 research study and relative effect sizes. All survey questions were 5-point Likert scale items where $1=$ strongly disagree, $2=$ disagree, $3=$ undecided, $4=$ agree, and 5=strongly agree. All $P<.001$.

\begin{tabular}{|c|c|c|c|c|c|}
\hline Specimen collection modality & $\mathrm{n}(\%)$ & Mean (SD) & Median (IQR) & Cohen $d$ & $95 \% \mathrm{CI}$ \\
\hline \multicolumn{6}{|l|}{ Willing to use on oneself $(\mathrm{N}=1435)$} \\
\hline Home: saliva sample & $1435(100.0)$ & $4.5(0.9)$ & $5(4-5)$ & Reference & $\mathrm{N} / \mathrm{A}^{\mathrm{a}}$ \\
\hline Home: throat swab & $1435(100.0)$ & $4.4(1.9)$ & $5(4-5)$ & 0.114 & 0.085 to 0.144 \\
\hline Home: blood sample & $1434(99.9)$ & $3.8(1.3)$ & $4(3-5)$ & 0.568 & 0.510 to 0.627 \\
\hline Drive-through site: throat swab & $1435(100.0)$ & $3.8(1.3)$ & $4(3-5)$ & 0.567 & 0.507 to 0.627 \\
\hline Clinic: throat swab & $1435(100.0)$ & $3.5(1.4)$ & $4(3-5)$ & 0.802 & 0.732 to 0.872 \\
\hline Clinic: blood sample & $1434(99.9)$ & $3.6(1.3)$ & $4(3-5)$ & 0.776 & 0.706 to 0.847 \\
\hline \multicolumn{6}{|c|}{ Willing to use on one's children ( $\mathrm{n}=385)$} \\
\hline Home: saliva sample & $385(100.0)$ & $4.1(1.3)$ & $5(4-5)$ & Reference & N/A \\
\hline Home: throat swab & $385(100.0)$ & $4.0(1.3)$ & $5(3-5)$ & 0.113 & -0.029 to 0.254 \\
\hline Home: blood sample & $334(87.8)$ & $3.4(1.5)$ & $3(2-5)$ & 0.454 & 0.388 to 0.520 \\
\hline Drive-through site: throat swab & $385(100.0)$ & $3.4(1.5)$ & $4(2-5)$ & 0.517 & 0.373 to 0.660 \\
\hline Clinic: throat swab & $385(100.0)$ & $3.1(1.5)$ & $3(2-5)$ & 0.742 & 0.596 to 0.889 \\
\hline Clinic: blood sample & $334(87.8)$ & $3.0(1.5)$ & $3(2-4)$ & 0.851 & 0.768 to 0.933 \\
\hline
\end{tabular}

${ }^{\mathrm{a}} \mathrm{N} / \mathrm{A}$ : not applicable.

\section{Discussion}

\section{Principal Findings}

Response to the SARS-CoV-2 epidemic in the United States has been hampered by insufficient testing both for diagnosing persons and for public health assessments to describe the epidemiology of infection. Critical shortages of reagents, other supply chain issues, and lack of availability of health care workers have led to gaps in testing, and it is critical to diversify testing methods. Options include alternative specimens to be tested and specimen collection locations. Further, it is important to understand which testing options are best suited to which purposes (eg, clinical care, population research, and screening versus diagnosis). Self-collection of specimens at home has proven to be an acceptable approach in other infectious disease testing, and it could play an important role in the response to the SARS-CoV-2 epidemic in the United States. 
Results from this study indicate that a large majority of adults would be willing to participate in a research study about SARS-CoV-2 infection or immune experience by collecting specimens at home and mailing them to a laboratory for testing. There was some preference for certain specimen collection modalities (saliva samples and throat swabs were preferred over blood samples); however, these differences were largely driven by preference for remote home specimen collection methods versus methods that would require visits to clinical or laboratory locations for testing. Testing location and specimen collection preferences were consistent across demographic groups and other characteristics of interest. These results are similar to literature reports that indicate that home specimen collection options are preferred to clinic-based testing methods for HIV and STI screening $[11,15,16]$.

These findings are important for the design of forthcoming epidemiologic studies of SARS-CoV-2 infection. Cross-sectional and cohort follow-up studies will depend on testing biological specimens to accurately measure disease prevalence, incidence, and recovery among participants. Our results indicate that study designs that use home specimen collection experience increased participation and higher retention compared to study designs that involve traveling to a drive-through site or clinic. The high willingness to use home specimen collection methods across demographic groups and other subgroups suggests that studies incorporating home specimen collection may be less susceptible to participation bias than designs requiring the collection of biological specimens in clinical settings.

The use of home specimen collection methods can also help ensure that research activities do not have a negative effect on adherence to current health guidance. Home specimen collection can be incorporated within the context text of social distancing guidelines [17], which can enable study participants to maximize their individual contributions to slowing the spread of COVID-19 disease. Similarly, reducing the contact between study participants and health care workers improves health care worker safety by reducing their risk of exposure. Further, such options can reduce the overall burden on health care providers and clinics that may not have the capacity to collect specimens as part of ongoing research. For instance, the current shortage of personal protective equipment among health care workers in the United States has been well documented [18], and the incorporation of home specimen collection can ensure that these resources are preserved for use in clinical care.

Willingness of people to use home specimen collection kits for research offers promising opportunities to conduct representative and timely research about SARS-CoV-2 infection and immune response. However, it is important to recognize that the collection of home specimens will also require rigorous testing to ensure that the laboratory results obtained provide accurate indications of SARS-CoV-2 infection and immune response, the kits are safe for participants to use, and the specimens are sufficiently robust to maintain validity after the process of return shipping to laboratories for analysis. It is important to distinguish between home collection of specimens that are mailed back to laboratories for analysis from the separate field of home testing, in which participants collect their own specimen, apply it to a test device, and interpret the results at home.

One reason that the use of home specimen collection may be associated with high willingness to participate in research related to SARS-CoV-2 infection is that the virus is highly infectious, and there is meaningful risk associated with entering clinical settings where people with symptoms of COVID-19 are congregated. Unlike testing as part of research studies for less contagious infectious diseases or for other types of disease, potential research participants who do not have symptoms may be particularly reluctant to report to clinical locations for screening. Relatedly, if home collection of specimens is developed and validated, it will be possible to conduct epidemiological studies that can both reach people in diverse geographic areas, including rural areas, and allow research to be conducted without exposing participants to potential harms associated with going to research sites that may result in their exposure to SARS-CoV-2.

\section{Limitations}

Our study results have several limitations. First, we assessed willingness using Likert scale items, which limits the ability to determine the magnitude of preference for one test modality over the other for any individual. However, Likert data are especially well suited for assessing the direction of preference, which is a relevant outcome given our desire to understand the potential impact of offering home specimen collection in research settings. Second, our recruitment methods targeted social media users, and our convenience sample may not be representative of all US adults. Third, opinions regarding testing for SARS-CoV-2 may change over time, given the rapidly shifting nature of public perception regarding the epidemic, and updates are merited to ensure that participant preferences remain stable. Finally, we know that there have historically been disconnects between expressed willingness to use self-testing or at-home specimen collection options for infectious diseases and the actual uptake of these highly acceptable devices [19]. However, compared to historical examples of the introduction of at-home tests before the advent of telemedicine, testing for SARS-CoV-2 infection with specimens collected at home for research or clinical purposes may be more acceptable given the broad availability of telehealth clinical services. These services may be used to provide support for participants who have questions about collecting specimens at home or to observe the self-collection of specimens at home until data are developed to document the sufficiency and quality of specimens collected at home.

\section{Conclusions}

Large scale population-based research and testing is needed to provide the epidemiologic data necessary to guide our public health response to the COVID-19 pandemic. Home specimen collection strategies should be considered to achieve the highest levels of participant engagement and retention, reduce the burden of specimen collection in overloaded health care settings, and reduce potential exposure of research participants to SARS-CoV-2 in research settings. 


\section{Acknowledgments}

This work was supported by the US National Institute of Allergy and Infectious Diseases (3R01AI143875-02S1) and the Emory Center for AIDS Research (P30AI050409). The content is solely the responsibility of the authors and does not necessarily represent the official views of the National Institutes of Health.

\section{Conflicts of Interest}

None declared.

\section{References}

1. Holshue ML, DeBolt C, Lindquist S, Lofy KH, Wiesman J, Bruce H, et al. First Case of 2019 Novel Coronavirus in the United States. N Engl J Med 2020 Mar 05;382(10):929-936. [doi: 10.1056/nejmoa2001191]

2. Coronavirus Disease 2019 (COVID-19): Cases in the U.S. US Centers for Disease Control and Prevention. URL: https:/ /www.cdc.gov/coronavirus/2019-ncov/cases-updates/cases-in-us.html [accessed 2020-04-08]

3. Our most up-to-date data and annotations. The COVID Tracking Project. URL: $\underline{\text { https://covidtracking.com/data [accessed }}$ 2020-04-08]

4. Guest J, Del Rio C, Sanchez T. The Three Steps Needed to End the COVID-19 Pandemic: Bold Public Health Leadership, Rapid Innovations, and Courageous Political Will. JMIR Public Health Surveill 2020 Apr 06;6(2):e19043 [FREE Full text] [doi: 10.2196/19043] [Medline: 32240972]

5. Spielberg F, Levine RO, Weaver M. Self-testing for HIV: a new option for HIV prevention? Lancet Infect Dis 2004 Oct;4(10):640-646. [doi: 10.1016/s1473-3099(04)01150-8]

6. Wiesenfeld HC, Lowry DL, Heine RP, Krohn MA, Bittner H, Kellinger K, et al. Self-collection of vaginal swabs for the detection of Chlamydia, gonorrhea, and trichomoniasis: opportunity to encourage sexually transmitted disease testing among adolescents. Sex Transm Dis 2001 Jun;28(6):321-325. [doi: 10.1097/00007435-200106000-00003] [Medline: 11403188]

7. Gilbert M, Hottes TS, Kerr T, Taylor D, Fairley CK, Lester R, et al. Factors associated with intention to use internet-based testing for sexually transmitted infections among men who have sex with men. J Med Internet Res 2013 Nov 14;15(11):e254 [FREE Full text] [doi: 10.2196/jmir.2888] [Medline: 24240644]

8. Hottes TS, Farrell J, Bondyra M, Haag D, Shoveller J, Gilbert M. Internet-based HIV and sexually transmitted infection testing in British Columbia, Canada: opinions and expectations of prospective clients. J Med Internet Res 2012 Mar 06;14(2):e41 [FREE Full text] [doi: 10.2196/jmir.1948] [Medline: 22394997]

9. Figueroa C, Johnson C, Ford N, Sands A, Dalal S, Meurant R, et al. Reliability of HIV rapid diagnostic tests for self-testing compared with testing by health-care workers: a systematic review and meta-analysis. Lancet HIV 2018 Jun;5(6):e277-e290. [doi: 10.1016/s2352-3018(18)30044-4]

10. Lunny C, Taylor D, Hoang L, Wong T, Gilbert M, Lester R, et al. Self-Collected versus Clinician-Collected Sampling for Chlamydia and Gonorrhea Screening: A Systemic Review and Meta-Analysis. PLoS One 2015;10(7):e0132776 [FREE Full text] [doi: 10.1371/journal.pone.0132776] [Medline: 26168051]

11. Siegler AJ, Mayer KH, Liu AY, Patel RR, Ahlschlager LM, Kraft CS, et al. Developing and Assessing the Feasibility of a Home-based Preexposure Prophylaxis Monitoring and Support Program. Clin Infect Dis 2019 Jan 18;68(3):501-504 [FREE Full text] [doi: 10.1093/cid/ciy529] [Medline: 29982304]

12. Sullivan GM, Artino AR. Analyzing and Interpreting Data From Likert-Type Scales. J Grad Med Educ 2013 Dec;5(4):541-542. [doi: 10.4300/jgme-5-4-18]

13. Carifio J, Perla R. Resolving the 50-year debate around using and misusing Likert scales. Med Educ 2008 Dec;42(12):1150-1152. [doi: 10.1111/j.1365-2923.2008.03172.x] [Medline: 19120943]

14. Cohen J. Statistical Power Analysis for the Behavioral Sciences 2nd Edition. New York, NY: Routledge; 2013.

15. Pearson WS, Kreisel K, Peterman TA, Zlotorzynska M, Dittus PJ, Habel MA, et al. Improving STD service delivery: Would American patients and providers use self-tests for gonorrhea and chlamydia? Prev Med 2018 Oct;115:26-30. [doi: 10.1016/j.ypmed.2018.08.007] [Medline: 30096329]

16. Odesanmi TY, Wasti SP, Odesanmi OS, Adegbola O, Oguntuase OO, Mahmood S. Comparative effectiveness and acceptability of home-based and clinic-based sampling methods for sexually transmissible infections screening in females aged 14-50 years: a systematic review and meta-analysis. Sex Health 2013;10(6):559. [doi: 10.1071/sh13029]

17. Coronavirus Disease (COVID-19): Social Distancing, Quarantine, and Isolation. US Centers for Disease Control and Prevention. URL: https://www.cdc.gov/coronavirus/2019-ncov/prevent-getting-sick/social-distancing.html [accessed 2020-04-12]

18. Kamerow D. Covid-19: the crisis of personal protective equipment in the US. BMJ 2020 Apr 03;369:m1367. [doi: 10.1136/bmj.m1367] [Medline: 32245847]

19. Colfax GN, Lehman JS, Bindman AB, Vittinghoff E, Vranizan K, Fleming PL, Multisite Evaluation of Surveillance of HIV (MESH) Study Group. What happened to home HIV test collection kits? Intent to use kits, actual use, and barriers to use among persons at risk for HIV infection. AIDS Care 2002 Oct;14(5):675-682. [doi: 10.1080/0954012021000005533a] [Medline: $\underline{\text { 12419117] }}$ 


\section{Abbreviations}

STI: sexually transmitted infection

Edited by G Eysenbach; submitted 19.04.20; peer-reviewed by S Badawy, C Figueroa, R McGowan; comments to author 20.06.20; revised version received 01.07.20; accepted 11.08.20; published 03.09.20

Please cite as:

Hall EW, Luisi N, Zlotorzynska M, Wilde G, Sullivan P, Sanchez T, Bradley H, Siegler AJ

Willingness to Use Home Collection Methods to Provide Specimens for SARS-CoV-2/COVID-19 Research: Survey Study

J Med Internet Res 2020;22(9):e19471

URL: https://www.jmir.org/2020/9/e19471

doi: $10.2196 / 19471$

PMID: $\underline{32790639}$

CEric William Hall, Nicole Luisi, Maria Zlotorzynska, Gretchen Wilde, Patrick Sullivan, Travis Sanchez, Heather Bradley, Aaron J Siegler. Originally published in the Journal of Medical Internet Research (http://www.jmir.org), 03.09.2020. This is an open-access article distributed under the terms of the Creative Commons Attribution License (https://creativecommons.org/licenses/by/4.0/), which permits unrestricted use, distribution, and reproduction in any medium, provided the original work, first published in the Journal of Medical Internet Research, is properly cited. The complete bibliographic information, a link to the original publication on http://www.jmir.org/, as well as this copyright and license information must be included. 